\title{
In Vivo Osteogenic Differentiation of Stem Cells Inside Compartmentalized Capsules Loaded with Co-cultured Endothelial Cells
}

Clara R. Correia ${ }^{1,2, \Psi}$, Tírcia C. Santos ${ }^{1,2}$, Rogério P. Pirraco ${ }^{1,2}$, Mariana T. Cerqueira ${ }^{1,2}$, Alexandra P. Marques $^{1,2}$, Rui L. Reis ${ }^{1,2}$, João F. Mano ${ }^{1,2, \psi, *}$

${ }^{1}$ 3B's Research Group - Biomaterials, Biodegradables and Biomimetics, University of Minho, Headquarters of the European Institute of Excellence on Tissue Engineering and Regenerative Medicine, AvePark, 4805-017 Barco, Guimarães, Portugal

${ }^{2}$ ICVS/3B's - PT Government Associate Laboratory, Braga/Guimarães, Portugal

${ }^{\psi}$ Present address: CICECO, Department of Chemistry, University of Aveiro, 3810-193 Aveiro, Portugal

*Corresponding author. Email: jmano@ua.pt

Keywords: adipose stem cells, co-culture, liquified capsules, microparticles, multilayers

\begin{abstract}
Capsules coated with polyelectrolytes and co-encapsulating adipose stem (ASCs) and endothelial (ECs) cells with surface modified microparticles are developed. Microparticles and cells are freely dispersed in a liquified core, responsible to maximize the diffusion of essential molecules and allowing the geometrical freedom for the autonomous three-dimensional (3D) organization of cells. While the membrane wraps all the instructive cargo elements within a single structure, the microparticles provide a solid 3D substrate for the encapsulated cells. Our hypothesis is that inside this isolated biomimetic 3D environment, ECs would lead ASCs to differentiate into the osteogenic lineage to ultimately generate a mineralized tissue in vivo. For that, capsules encapsulating only ASCs (MONO capsules) or co-cultured with ECs (CO capsules) are subcutaneously implanted in nude mice up to 6 weeks. Capsules implanted immediately after production or after 21 days of in vitro osteogenic stimulation are tested. The most valuable outcome of the present study is the
\end{abstract}


mineralized tissue in $\mathrm{CO}$ capsules without in vitro pre-differentiation, with similar levels compared to the pre-stimulated capsules in vitro. We believe that the proposed bioencapsulation strategy is a potent self-regulated system, which might find great applicability in bone tissue engineering.

\section{Introduction}

The regeneration of large bone defects remains a major concern in the orthopaedic field. To avoid the potential disadvantages of autografts and allografts, namely their limited supply and disease transmission potential [1], engineered bone constructs have been proposed as promising alternatives in clinical applications. Donor-site morbidity associated to cell isolation (both osteoblasts or bone marrow-derived mesenchymal stem cells) and limited proliferative capacity of primary osteoblasts are major challenges in providing adequate numbers for transplantation $[2,3]$. In addition to bone marrow, adipose tissue has been identified as a source of multipotent cells that due to the capacity to differentiate into adipogenic, chondrogenic, myogenic, and osteogenic lineages are seen as promising for tissue regeneration [4,5]. Moreover, adipose tissue is often available in an abundant quantity after a relatively easy harvesting. However, controlling mesenchymal differentiation and engineering bone in vivo are challenging accomplishments, as it often leads to heterotypic and inferior osseous tissues. Additionally, when osteogenesis is aimed at, angiogenesis must be considered since it plays an important role during the development and maturation of bone $[6,7]$. The induction of orchestrated osteo- and angiogenesis by biochemical stimulation $[8,9]$ or by co-culturing osteoprogenitor and endothelial cells [10-13] has been shown to better induce bone tissue formation, as well as to improve survival of the engineered tissues. Particularly, considering native bone vascular network, osteoprogenitor cells inevitably interact with endothelial cells (ECs) in a synergetic fashion $[14,15]$. While endothelial cells secrete numerous regulatory molecules that influence the differentiation and activity of bone forming cells [16-19], osteoprogenitor cells release diverse pro-angiogenic growth factors $[11,13,20,21]$. To mimic such concept, one could envisage the in vitro development of a single reservoir that contain instructive signals to, upon implantation, generate bone-like tissues. In this context, we propose a novel bioencapsulation strategy whose in vivo performance is assessed for the first time. With this work we expect to highlight the potential of 
liquified and multilayered capsules as an alternative methodology to the commonly used 3D porous structures/hydrogels for TE. For that, mesenchymal stem and endothelial cells were both isolated from the adipose tissue and co-cultured within liquified and multilayered capsules. Following multiscale assembling strategies [22], the capsules are composed by three essential components: (i) a permselective nanostructured membrane that wraps the liquefied core of the capsule ensuring permeability to essential molecules for cell survival, and the creation of a single reservoir to facilitate the implantation procedure of multiple components; (ii) poly(L-lactic acid) (PLLA) microparticles surface functionalized with plasma treatment and collagen I that create a 3D microenvironment for the encapsulated cell and act as cell adhesion sites; and (iii) adipose stem (ASCs) and endothelial (ECs) cells. The membrane was produced by layer-by-layer ( $\mathrm{LbL}$ ) technique, which is a versatile, low-cost technique, and more importantly for bioencapsulation systems, can be performed under mild conditions $[23,24]$. We hypothesized that within the controlled environment of capsules, endothelial cells would promote the osteogenic differentiation of ASCs due to their specific crosstalk, leading to the formation of a mineralized tissue in vivo. The design of the experiment is represented in Scheme 1. Capsules containing only ASCs (MONO capsules) or ASCs and ECs (CO capsules) were produced 21 days before the implantation (day -21 ), and subsequently cultured in osteogenic differentiation medium to stimulate in vitro the differentiation of the ASCs. At the day of implantation (day 0), the production of MONO and CO capsules was repeated. Additionally, capsules without encapsulated cells (MATERIAL) were also produced. All the produced capsules, i.e. after 21 days of in vitro osteogenic stimulation or freshly prepared, were implanted up to 6 weeks in nude mice, each one with two craniolateral-oriented pockets subcutaneously created by blunt dissection. Three capsules of the same formulation were implanted at each pocket (Scheme 1A). At predetermined intervals, the implants, which could be macroscopically identified (Scheme 1B), were retrieved for histological analysis. 


\section{Materials and methods}

\subsection{Cells isolation and characterization}

Subcutaneous adipose tissue from liposuction procedures was used to isolate both human ASCs [25] and human ECs [26]. The collected tissues were obtained under a cooperation agreement between the 3B's Research Group and Hospital da Prelada (Porto, Portugal), after approval of the respective ethical committees. Patient's informed consent and anonymity were assured. Samples were transported in phosphate-buffered saline (PBS, Sigma-Aldrich) supplemented with penicillinstreptomycin (10\%, pen-strep, Gibco) and kept at $4^{\circ} \mathrm{C}$ for a maximum of $24 \mathrm{~h}$. Lipoaspirates were washed with PBS and incubated with collagenase type II A (0.05\% w/v, Sigma-Aldrich) for 45 minutes at $37^{\circ} \mathrm{C}$ in a shaking water bath. The digested samples were filtered $(200 \mu \mathrm{m})$ and centrifuged (800 g, $\left.10 \mathrm{~min}, 4^{\circ} \mathrm{C}\right)$. The obtained stromal vascular fraction (SVF) was resuspended in erythrocyte lysis buffer ( $\mathrm{pH}$ 7.4) containing ammonium chloride (155 mM, Merk), potassium bicarbonate (5.7 mM, Riedel-de-Häen), and ethylenediaminetetraacetic acid (0.1 M, EDTA, SigmaAldrich) in distilled water. After $10 \mathrm{~min}$ of incubation at room temperature (RT), the mixture was centrifuged (300 g, $5 \mathrm{~min}$ ). To obtain the ASCs cultures, the red blood cell-free SVF was resuspended in a-MEM medium (Invitrogen) supplemented with fetal bovine serum (10\%, FBS, Invitrogen) and pen-strep ( $1 \% \mathrm{v} / \mathrm{v})$. The ECs cultures were obtained from the red blood cell-free SVF that was resuspended in EndoGro ${ }^{\mathrm{TM}}$-MV-VEGF complete media kit (Millipore lberica). ECs were plated in cell culture flasks, previously coated with gelatin $(0.7 \% \mathrm{w} / \mathrm{v}$, porcine skin type $A$, Sigma-Aldrich) for $30 \mathrm{~min}$ at $37^{\circ} \mathrm{C}$. Culture medium was changed $48 \mathrm{~h}$ after initial plating and then every 3-4 days for both cell phenotypes.

The phenotypic profile of the isolated ASCs and ECs was assessed by a flow cytometer (BD FACSCalibur, CellQuest v3.3 software, BD Biosciences). Mesenchymal (CD105-FITC, Arium; CD90-APC, and CD73-PE, BD Biosciences), endothelial (CD31-APC, Citomed) and hematopoietic (CD34-PE, BD Biosciences) markers were quantified. Briefly, the isolated cells were harvested by TrypLE $^{\text {TM }}$ Express solution (Life Technologies) at $37^{\circ} \mathrm{C}$ for $5 \mathrm{~min}$, and centrifuged. Samples were resuspended in PBS solution containing bovine serum albumin (3\% w/v, BSA, Sigma-Aldrich) and the specified antibodies at the dilutions defined by the manufacture's specifications. After 20 min at 
RT, samples were subsequently washed with PBS, centrifuged, fixed in formaldehyde $(1 \% \mathrm{v} / \mathrm{v}$, Sigma-Aldrich) for analysis. More than $90 \%$ of the isolated ASCs were positive for all the mesenchymal stem cells markers tested, whilst lacking the endothelial marker CD31. Additionally, ASCs expression for CD34 was bellow $2 \%$. On the other hand, for ECs the expression of the endothelial marker CD31 was $98 \%$ and for CD34 around $2 \%$.

\subsection{Microparticles production and surface functionalization}

Poly(L-lactic acid) microparticles ( $\mu$ PLLAs) were produced by an oil/water (o/w) emulsion technique and surface modified with collagen I after plasma treatment as similarly described in our previous reports [27-30]. Briefly, PLLA (5\% w/v, Mw 1,600-2,400, 70\% crystallinity, Polysciences) was dissolved in methylene chloride $\left(\mathrm{CH}_{2} \mathrm{Cl}_{2}\right.$, Fisher Chemical). Under agitation, this solution was added to polyvinyl alcohol (0.5\%, PVA, Sigma-Aldrich). After 2 days at RT, the produced $\mu$ PLLAs were subsequently collected, washed with distilled water, and lyophilized (Cryodos, Telstar) for 3 days. Microparticles were then placed in a plasma reactor chamber (PlasmaPrep5, Gala Instrumente) fitted with a radio frequency generator. Air was used as the working atmosphere. A glow discharge plasma (0.2 mbar, 30V, $15 \mathrm{~min})$ was created. Subsequently, microparticles $(500 \mathrm{mg})$ were immersed for $4 \mathrm{~h}$ at $\mathrm{RT}$ in collagen I solution (1200 $\mu \mathrm{g}$, rat protein tail, Gibco).

\subsection{Mono- and co-cultures set up}

Sodium alginate $(1.5 \% \mathrm{w} / \mathrm{v}, \mathrm{ALG}, \sim 250 \mathrm{cP}$, low viscosity from brown algae, Sigma-Aldrich) was dissolved in sodium chloride $(0.15 \mathrm{M}, \mathrm{NaCl}$, Enzymatic) buffered at $\mathrm{pH} 7$ with MES hydrate $(25 \mathrm{mM}$, Sigma-Aldrich). The solution was sterilized by $0.22 \mu \mathrm{m}$ filtration prior mixing with the cells. At $90 \%$ confluence, ASCs (passage 2) and ECs (passage 4-6) were detached using TrypLE ${ }^{\text {TM }}$ Express solution (Life Technologies) at $37^{\circ} \mathrm{C}$ for $5 \mathrm{~min}$. PBS was added and the cell suspensions were centrifuged for $5 \mathrm{~min}$ at $300 \mathrm{~g}$. Cells (ASCs alone or mixed with ECs, 1:1 ratio) resuspended in the alginate solution $\left(5 \times 10^{6}\right.$ cells per $\mathrm{mL}$ of alginate) were added to the microparticles $\left(50 \mathrm{mg} \cdot \mathrm{mL}^{-1}\right.$ of alginate) and then used to produce liquified capsules containing ASCs alone (MONO capsules) or in co-culture with ECs (CO capsules). 


\subsection{Liquified multilayered capsules production}

All the conditions used to produce liquified capsules (the combination of the polyelectrolytes, concentration, $\mathrm{pH}$, number of layers, and time of adsorption and liquefaction) were optimized in our previous studies [27-30]. Briefly, alginate solutions containing cells were added drop wise using a $21 \mathrm{G}$ needle to a calcium chloride solution $\left(0.1 \mathrm{M}, \mathrm{CaCl}_{2}, \mathrm{VWR}\right)$ buffered at $\mathrm{pH} 7$ with MES hydrate (25 mM, Sigma-Aldrich). After 20 min at RT, alginate hydrogel particles were collected and rinsed in a washing solution of $\mathrm{NaCl}(0.15 \mathrm{M})$. The external membrane was processed by layer-by-layer adsorption. Hydrogel particles were subsequently immersed in poly-L-lysine (PLL, $M_{w} \sim 30,000$ 70,000, pH 7, Sigma-Aldrich), ALG solution ( $\mathrm{pH} 7$ ), chitosan (CHT, $\mathrm{pH} \mathrm{6,} \mathrm{water-soluble} \mathrm{highly}$ purified, viscosity $107 \mathrm{mPa} . \mathrm{s}, \mathrm{M}_{\mathrm{w}}=2.7 \times 10^{5} \mathrm{~g} \cdot \mathrm{mol}^{-1}$, 83\% degree of deacetylation, Protasan UP CL 213, NovaMatrix), and, ultimately, in ALG solution again. All the polyelectrolyte solutions (0.5 $\left.\mathrm{mg} \cdot \mathrm{mL}^{-1}\right)$ were prepared in $\mathrm{NaCl}(0.15 \mathrm{M})$ containing MES hydrate $(25 \mathrm{mM})$. Following a $10 \mathrm{~min}$ adsorption period for each polymer, the excess of macromolecules was removed by immersion in $\mathrm{NaCl}(0.15 \mathrm{M})$ for $5 \mathrm{~min}$. This process was repeated three times in order to obtain a 12-layered membrane. Coated hydrogel particles were immersed in EDTA $(20 \mathrm{mM})$ at pH 7 for 5 min to liquefy the alginate core. To induce osteogenic differentiation in vitro, MONO and CO capsules were cultured for 21 days in supplemented medium composed by complete EndoGro ${ }^{\text {TM}}$-MV-VEGF medium supplemented with $\beta$-glycerophosphate (10 mM, Sigma-Aldrich), ascorbic acid (50 $\left.\mu \mathrm{g} \cdot \mathrm{mL}^{-1}\right)$, and dexamethasone (10 nM, Sigma-Aldrich).

\subsection{Lipophilic fluorescent labelling}

Prior encapsulation, ASCs and ECs were incubated with lipophilic dyes, namely 3,3' dioctadecyloxacarbocyanine perchlorate (DIO, green) and 1,1'-dioctadecyl-3,3,3',3'tetramethylindocarbocyanine perchlorate (DIL, red), respectively. Cells were incubated with each dye $\left(1 \mathrm{~mL}, 2 \mu \mathrm{M}\right.$ per $1 \times 10^{6}$ cells) at $37^{\circ} \mathrm{C}$ for $10 \mathrm{~min}$. Then, MONO and CO capsules were produced as previously described encapsulating fluorescently labelled cells. Capsules were visualized by confocal microscopy (TCS SP8, Leica). 


\subsection{DNA and alkaline phosphatase activity quantification}

MONO and CO capsules cultured in osteogenic differentiation medium for 21 days were analysed after cell lysis for DNA amount and alkaline phosphatase activity (ALP). Four capsules per well were suspended in $1 \mathrm{~mL}$ of ultra-pure water. After $1 \mathrm{~h}$ in a $37^{\circ} \mathrm{C}$ shaking water bath, the solutions were frozen at $-80^{\circ} \mathrm{C}$ overnight. Samples were defrosted and used according to the specifications of the kit (Quant-iT ${ }^{\mathrm{TM}}$ PicoGreen ${ }^{\circledR}$ dsDNA assay kit, Life Technologies). A standard curve was generated with the provided dsDNA solution. After $10 \mathrm{~min}$ of incubation at RT, fluorescence was read at an excitation wavelength of $485 / 20 \mathrm{~nm}$ and $528 / 20 \mathrm{~nm}$ of emission using a microplate reader (Gen 5 2.01, Synergy HT, Biotek). Then, the activity of ALP in the remaining solutions was quantified. Briefly, a substrate solution ( $\mathrm{pH}$ 9.8) was prepared by dissolving 4-nitrophenylphosphate disodium salt hexahydrate $(0.2 \% \mathrm{w} / \mathrm{v}$, Sigma-Aldrich) in diethanolamine ( $1 \mathrm{M}$, Sigma-Aldrich). Each sample $(20 \mu \mathrm{L})$ was mixed with the prepared substrate solution $(60 \mu \mathrm{L})$. After $45 \mathrm{~min}$ at $37^{\circ} \mathrm{C}$ protected from light, the reaction was stopped with $80 \mu \mathrm{L}$ of a $\mathrm{NaOH}(2 \mathrm{M})$ and EDTA $(0.2 \mathrm{mM})$ solution. A standard curve was prepared with diluted series of 4-nitrophenol solution (10 mM, Sigma-Aldrich). Absorbance was read at $405 \mathrm{~nm}$ in a microplate reader (Gen 5 2.01, Synergy HT, Biotek). ALP activity results were normalized with dsDNA quantification data of MONO capsules.

\subsection{Mineralization analysis}

MONO and CO capsules cultured in osteogenic differentiation medium for 21 days were washed in PBS, and processed in an automatic spin tissue processor (STP120-2, Microm) for histological analysis. Samples were embedded in paraffin and cut into sections of $5 \mu \mathrm{m}$ thickness using a microtome (HM355S, Microm). To visualize calcium-rich deposits, sections were deparaffinized and subsequently immersed in alizarin red staining solution for $5 \mathrm{~min}$ at RT. Samples were then mounted and analysed by microscopy (Axio Imager Z1m, Zeiss).

\subsection{Animals surgery, euthanasia, and implants retrieval}

The animal experimentation project was approved by the institutional Animal Welfare Body (SECVS 032/2015) and the National Authority Direção Geral de Alimentação e Veterinária. Five week-old 
male nude mice (average weight of $25 \mathrm{~g}$ ) were used to subcutaneously implant MONO and CO capsules, either freshly prepared or after 21 days in vitro in osteogenic differentiation medium, and capsules without encapsulated cells (MATERIAL). Four mice were used for each formulation and at each timepoint $(1,3$, and 6 weeks). Under surgical sterile conditions, animals were anaesthetized with an intraperitoneal injection of a mixture of ketamine (75 mg.kg ${ }^{-1}$, Imalgene ${ }^{\circledR}$, Merial) and metedomidine (1 mg. $\mathrm{kg}^{-1}$, Domitor ${ }^{\circledR}$, Orion). One medial incision of approximately $0.7 \mathrm{~cm}$ was performed in the dorsum of the mice and two craniolateral-oriented pockets were subcutaneously created by blunt dissection. Three capsules were inserted into each pocket. Pockets without capsules were also used as control (EMPTY). Subsequently, the panniculus carnosus and the skin were carefully sutured with non-resorbable $4 / 0$ sutures. After the surgical procedure, the animals were induced to recover from anaesthesia with an intraperitoneal injection of Atipazemol (1 mg. $\mathrm{kg}^{-1}$, Antisedan, Orion). The animals were kept housed together (different conditions in each cage) with standard diet and water ad libitum during all the time of the experiment. After 1, 3, and 6 weeks of implantation, the animals were euthanized by carbon dioxide inhalation. The implanted capsules and the respective surrounding tissue were explanted from each animal. The collected explants were subjected to macroscopic observation and fixed in $10 \%$ formalin for $24 \mathrm{~h}$ at RT. Samples were then processed for standard histological evaluation.

\subsection{In vivo histological analysis}

The explants were processed in an automatic ethanol-xylene spin tissue processor (STP120-2, Microm) and then embedded in paraffin. Histological sections $(5 \mu \mathrm{m})$ were obtained using a microtome (HM355S, Microm, ThermoFisher Scientific). Before each histological staining, the sections were automatically deparaffinized and rehydrated (HMS740, Microm, ThermoFisher Scientific). After each staining protocol, unless otherwise specified, the sections were immediately dehydrated and immersed in xylene, before being mounted (Entellan, Millipore). Ultimately, the stained sections were visualized with Axio Imager Z1m (Zeiss). 


\subsubsection{Hematoxylin and eosin (H\&E) staining}

The samples for H\&E staining protocol were automatically processed (HMS740, Microm, ThermoFisher Scientific). Briefly, histological sections were subsequently immersed in hematoxylin (BioOptica) and after bluing in an ammonia solution ( $1 \% \mathrm{v} / \mathrm{v})$, in eosin y (BioOptica).

\subsubsection{CD31 immunohistochemistry}

Antigen retrieval was performed in heat-mediated $\left(95-100^{\circ} \mathrm{C}\right)$ sodium citrate buffer $(10 \mathrm{mM}$, SigmaAldrich) for $20 \mathrm{~min}$. After cool down for $20 \mathrm{~min}$, endogenous peroxidases were inactivated with hydrogen peroxide (3\% v/v, AnalaR Normapur) and non-specific binding blocked with horse serum incubation (2.5\% NHS, Vectastain Universal Quick Kit, Vector Laboratories) for $30 \mathrm{~min}$ at RT. The sections were incubated with the primary antibody rabbit polyclonal anti-human to CD31 (1:50 diluted in PBS with NHS, Abcam) in a humidified chamber at $4^{\circ} \mathrm{C}$ overnight. The second antibody (Vectastain Universal Quick Kit, Vector Laboratories) was added for $1 \mathrm{~h}$ at RT, and the stained revealed with a DAB solution prepared according to manufacturer's specifications (Vectastain Universal Quick Kit, Vector Laboratories). Sections were counterstained with hematoxylin (BioOptica) before microscopic analysis.

\subsubsection{Masson's trichrome staining}

Sections were stained for $5 \mathrm{~min}$ in a solution containing azure B $(0.5 \mathrm{~g}$, Sigma-Aldrich), ammonium iron (5 g, ammonium iron sulfate (III) dodecahydrate, Millipore) and distilled water (100 $\mathrm{mL}$ ). Sections were then subsequently stained with hematoxylin (BioOptica) and picric ethanol (1\% w/v, Sigma-Aldrich) for $5 \mathrm{~min}$ each. After $10 \mathrm{~min}$ washing in running tap water, sections were stained with the solutions from the Trichrome Stain Masson kit (Sigma-Aldrich) according to the manufacturer's specifications.

\subsubsection{Osteopontin immunofluorescence}

The antigen retrieval was performed in a heat-mediated sodium citrate buffer $(10 \mathrm{mM}$, SigmaAldrich). Non-specific binding was blocked by immersion in BSA ( $3 \% \mathrm{w} / \mathrm{v})$ for $30 \mathrm{~min}$ at RT. 
Samples were then incubated overnight at $4^{\circ} \mathrm{C}$ with the primary antibody rabbit anti-human osteopontin (1:100 in 1\% BSA, Abcam). After a PBS washing, samples were incubated for $1 \mathrm{~h}$ at RT with the secondary antibody anti-rabbit AlexaFluor 488 (1:500 in 1\% BSA, Molecular Probes). Samples incubated only with the secondary antibody were used as controls. Ultimately, samples were subsequently counterstained with DAPI (1 mg. $\mathrm{mL}^{-1}$ diluted 1:1000 in PBS, Sigma-Aldrich) for 5 min at RT, immediately mounted (Permafluor, ThermoFisher Scientific), and analysed by fluorescence microscopy (Axio Imager Z1m, Zeiss). The percentage of green intensity was measured by Image $J$ software (version 1.440). Each image $(n=4)$ was split in the three RGB channels. In the green channel $(G)$ the intensity of fluorescence was quantified with a threshold of 45-255.

Alizarin Red Staining: To assess the presence of calcium-rich deposits (red), the sections were immersed in alizarin red solution ( $2 \% \mathrm{w} / \mathrm{v}$, Sigma-Aldrich) for $5 \mathrm{~min}$ at RT. The percentage of red intensity was measured by Image $J$ software (version 1.440). Each image $(n=4)$ was split in the three RGB channels. In the red channel $(R)$ the intensity of colour was quantified with a threshold of 0-206.

\subsection{Statistical analysis}

All the results were expressed as mean \pm standard deviation. Significant differences between the different groups were identified by one-way ANOVA using the post-hoc Tukey's with multiple comparison tests.

\section{Results}

\subsection{In vitro evaluation of the capsules}

Prior implantation, ASCs and ECs grown in 2D cell culture flasks were fluorescently marked with lipophilic dyes. The fluorescently marked cells were then encapsulated in MONO (Figure 1A) and $\mathrm{CO}$ (Figure 1B) capsules. Samples were immediately visualized by confocal microscopy. In MONO capsules, only green fluorescence corresponding to ASCs presence could be detected, while in CO 
capsules both green and red fluorescence were detected, identifying respectively ASCs and ECs. The dyes assay allowed to visualize the random distribution of ASCs and ECs encapsulated within the capsules liquified environment. Cells were only labelled prior to encapsulation for the lipophilic fluorescence assay.

After 21 days of culture in osteogenic differentiation medium, the secretion of the late osteogenic marker osteopontin (OP) was confirmed in MONO (Figure 1C) and CO capsules (Figure 1D). OP is a biomarker of mature osteoblast phenotypical behaviour, being widely used in osteogenic differentiation strategies [31,32]. It is a multifunctional extracellular glycoprotein primarily involved in the regulation of mineral deposition [33]. Among the non-collagenous matrix proteins present in bone, OP acts as a unique interfacial adhesion molecule, thus being responsible to maintaining the structural integrity of bone and bone/implant systems [34,35].

Additionally, mineralization was qualitatively assessed by alizarin red staining (Figure 1E and F), confirming the deposition of calcium. Ultimately, capsules were tested for DNA quantification (Figure 1G) and ALP activity (Figure 1H). As expected, day 0 capsules have the lowest DNA amount compared to day 21 capsules. This indicates that the encapsulated cells in day 21 capsules have proliferated during the in vitro culture. Moreover, the efficient encapsulation of the cells was proven by the double amount of DNA detected in the CO capsules that corresponds to the additional and equal amount of endothelial cells used. CO capsules after 21 days of culture continued to present the highest DNA amount, which seems to indicate the absence of deleterious effects of the system. Figure $\mathbf{1 H}$ illustrates the ALP activity normalized per $\mu \mathrm{g}$ DNA of the MONO capsules at day 0 and after 21 days of in vitro culture. Again, day 0 capsules have a significant lower ALP activity compared to day 21 capsules since the latter were cultured in vitro in supplemented medium to stimulate the osteogenic differentiation of the ASCs. Importantly, CO capsules cultured for 21 days presented the highest ALP activity compared to MONO capsules. 


\subsection{In vivo histological assessment}

\subsubsection{Morphological analysis}

Histological analysis of explanted samples stained with H\&E (Figure 2) showed good integration of all types of capsules within the surrounding host tissue. At the first week of implantation, the fragmentation of the layer-by-layer membrane of the capsules was visible. It seems that the disruption of the membrane occurred gradually since the encapsulation contents, composed by cells and microparticles, remained grouped at the site of the implantation. In addition, the histological evaluation with H\&E staining also revealed the presence of various blood vessels in MONO and CO capsules, while in MATERIAL capsules only few vessels could be observed. In the empty control, the presence of blood vessels could not be detected.

After 6 weeks of implantation, immunostaining of CD31 (Figure 3) was performed in MONO and CO capsule. As evidenced, the capsules were permeated to blood vessels. However, the CD31 was not specific for the endothelium. Particularly in the CO capsules, more areas with non-specific endothelium staining could be detected along the sections, which jeopardizes the visualization of the permeable blood vessels expressing CD31. This could be attributed to the fact that the coencapsulated endothelial cells are also expressing CD31. The presence of collagen was then assessed by Masson's Trichrome staining. As evidenced in Figure 4, the presence of collagen (blue staining) could be visualized mostly in MONO and CO capsules with 21 days of previous in vitro culture. Notably, although in less quantify, the presence of collagen in the newly deposited ECM could also be detected in CO capsules without in vitro stimulation. Additionally, the staining also allowed visualizing the presence of blood vessels with intraluminal red blood cells inside the capsules environment. Importantly, the presence of a dense layer of fibrotic connective tissue surrounding the capsules could not be visualized, which highlights the biotolerability of the capsules.

\subsubsection{Osteogenesis evaluation}

The osteogenic marker OP was qualitatively detected by fluorescence microscopy (Figure 5). As expected, OP was expressed within in vitro pre-differentiated MONO and CO capsules (day 21 
capsules). At the first week of implantation, OP could be earlier detected in pre-differentiated CO capsules. Latter, with increasing implantation time, the expression of OP could also be detectable in CO capsules without in vitro osteogenesis stimulation (day 0 capsules). The detection of OP with increased implantation time indicates the commitment of the encapsulated stem cells towards the osteogenic lineage. Only scarce fluorescence could be found in the material capsules as well as in day 0 MONO capsules formulations, indicating that osteogenic differentiation did not occur. Corroborating the OP results, mineralization (Figure 6) occurred in the pre-differentiated MONO and CO capsules, as well as in CO capsules without in vitro osteogenesis (day 0 capsules). Mineralization increased with the implantation time, and after 6 weeks of implantation calcium-rich deposits (stained in red) covered the entire region of the capsules. In the MONO capsules without pre-differentiation only a few mineralization spots were detected, while it was absence in the material capsules.

\section{Discussion}

In the current study, we demonstrate for the first time the in vivo osteogenic differentiation of stem cells driven by co-culture with endothelial cells within liquified and multilayered capsules containing solid microparticles.

Among the different bioencapsulation systems proposed [36-40], the central issue is related with the diffusion of nutrients to prevent necrosis at the bulk of implanted materials. In fact, the consensus in all tissue engineering fields is that improved vascularization is the crux of all future scaffold designs $[6,7,41]$. Particularly in bone tissue engineering, engineered scaffolds transplanted to patients to restore or improve the functions of damaged tissue have often failed to produce the desired results due to a deficient oxygen and nutrient diffusion. In order to address this issue, we developed a bioencapsulation system, in which the core is in a liquid state, hence maximizing mass transportation within all the regions of the construct. However, the majority of cells used in tissue engineering are anchorage dependent, and consequently a physical support to process different cellular metabolisms is required. Therefore, along with the encapsulated cells, solid microparticles were incorporated inside the liquified environment of the capsules, which act as cell adhesion sites. 
Additionally, microparticles also allowed creating a biomimetic 3D environment inside the capsules. Surrounding the liquified core loaded with the encapsulated cells and microparticles is the layer-bylayer membrane. As shown, the thin multilayer remained stable during 21 days of in vitro culture, and allowed the required diffusion of essential molecules for cell survival. The membrane also allowed the diffusion of osteogenic differentiation factors added to the culture medium that led to in vitro pre-osteogenic stimulation of MONO and CO capsules. The presence of the membrane was also essential to assemble all the encapsulated components into a single reservoir. Having a single structure encapsulating all the different components facilitated the implantation process, while avoiding their dispersion to other regions of the body. However, notwithstanding the stability shown in vitro, after the first week of implantation the histological analysis of the capsules revealed that the membrane was disrupted. Nevertheless, since the disruption of the membrane occurred only at some areas, the core contents of the capsules remained mobilized and aggregated at the site of implantation. The partial disruption could be explained by the fact that the capsules were implanted subcutaneously into created pockets, so they were under a squeeze effect between the host tissues. Additionally, as time increases, the presence of blood vessels could have also contributed to the disruption of the flexible membrane that together with the liquified core of capsules could not offer a rigid surface. However, the disruption of the membrane proved to be a great outcome since the blood vessels transport oxygen and nutrients necessary to the transplanted cells and to the tissue surrounding the implanted capsules. Our main hypothesis was that the co-encapsulation of endothelial cells with stem cells within the capsules environment would lead stem cells to differentiate into the osteogenic lineage and, ultimately, led to the formation of a mineralized tissue. Different fundamental studies [42-44] have been show that ASCs stimulate blood vessel growth due to the secretion of many pro-angiogenic factors, such as VEGF $[11,13,21]$. This explains the abundant presence of blood vessels in all capsules. Furthermore, endothelial cells also secrete numerous regulatory molecules for the differentiation and activity of bone forming cells [16-19]. However, in vivo this interaction is poorly characterized mainly because to the absence of appropriate engineered systems. Facca et al. [45] reported that although in vitro no substrate was required, the osteogenic differentiation of embryonic stem cells after in vivo implantation with 
microparticles incorporating BMP-2 and VEGF only occurred after incorporation of the contents in an alginate gel as a substrate. This was mainly attributed to the fact that the alginate matrix improved contact between the cells and the microparticles releasing system. In our strategy, the encapsulated stem and endothelial cells remained grouped due to the capsules structure. We believe that this has directly influenced the reported in vivo outcome by promoting a higher exposure to pro-angiogenic and osteogenic factors released by stem and endothelial cells, respectively. This interaction explains the significant advance of this work, namely the in vivo osteogenesis with a mineralized and vascularized matrix observed in the CO capsules implanted immediately after production. This is an interesting outcome, indicating that CO capsules might be readily implanted after production into a bone defect since osteogenesis occurred in vivo without requiring the standard in vitro stimulation of 21 days in differentiation medium. The co-culture contribution is supported by the absence of mineralized tissue in the MONO capsules also implanted immediately after production. The pre-differentiation strategy of MONO and CO capsules during 21 days in vitro in culture medium containing osteogenic differentiation factors also demonstrated that the osteogenic differentiation pattern was maintained in vivo. However, the predifferentiation of $\mathrm{CO}$ capsules, since endothelial cells are co-encapsulated, might result in a prevascularization in vitro. In bone TE, however, the in vivo success of in vitro prevascularization has so far been limited. Rouwkema et al. [46] have shown that vascular structures obtained from co-cultures of human umbilical vein endothelial cells (HUVECs) and human mesenchymal stem cells (hMSCs) were stable and organized into a more mature vascularization network after implantation. However, anastomosis of the invading vessels to the in vitro created vasculature was limited. Therefore, a vascularized osteogenic system that does not require in vitro culture before implantation is of great importance in TE strategies.

The present study also allowed reinforcing the potential of the proposed capsules for bone regeneration, as proposed in our previous in vitro study [29]. In the referred in vitro study, it was demonstrated that the co-encapsulation of ECs lead ASCs to differentiate into the osteogenic lineage inside the liquified and multilayered capsules with microparticles, even in the absence of two major osteogenic differentiation factors, namely dexamethasone and ascorbic acid. Additionally, it 
was demonstrated that CO capsules released BMP-2 and VEGF, which evidences the potential of the capsules as a cytokine delivery system. In the present study, we could confirm the potential of capsules for the first time in vivo without any in vitro stimulation.

In future work, it is our intention to fully validate their potential in a bone defect using immunocompetent models such as humanized models, and thus to study in more detail the relevance of the system in a clinical approach.

\section{Conclusion}

In this study, MONO and CO capsules with or without in vitro osteogenic pre-differentiation were subcutaneously implanted in nude mice up to 6 weeks. The in vivo implantation of capsules did not elicit a severe infiltration of inflammatory cells, demonstrating the biotolerability of the liquified capsules. Sign of necrosis, either in the host tissue surrounding the implanted capsules or in the newly formed tissue inside the capsules, could not be detected, which is a major concern when implanting 3D systems. The significant advance of this work is that the co-encapsulation of endothelial cells with stem cells led to the formation of a mineralized tissue in vivo. This work is also a unique demonstration of the in vivo performance of a liquified and multilayered capsule containing microparticles and a co-culture of endothelial and stem cells. Considering the reported findings, we suggest the use of $\mathrm{CO}$ capsules as self-regulated reservoirs that after production can be readily implanted. By using this formulation we can incorporate different kinds of active molecules aimed at different applications, such as gene therapy, drug delivery, or tissue engineering. We believe that the developed capsules will find great applicability in bone regeneration, but also in other tissue engineering domains of complex tissue restoration.

\section{Acknowledgements}

The authors acknowledge the financial support by the Portuguese Foundation for Science and Technology (FCT) through the Ph.D. (SFRH/BD/69529/2010-Clara R. Correia) and the Post-doc (SFRH/BPD/96611/2013-Mariana T. Cerqueira) grants, and the funding of RL3-TECT-NORTE-01- 
0124-FEDER-000020 for Rogério P. Pirraco. The authors are also grateful to Dr. Teresa Oliveira for her valuable help with the tissue processing and histological procedures.

\section{References}

[1] K.S. Griffin, K.M. Davis, T.O. McKinley, J.O. Anglen, T.-M.G. Chu, J.D. Boerckel, M.A. Kacena, Evolution of bone grafting: bone grafts and tissue engineering strategies for vascularized bone regeneration, Clinical Reviews in Bone and Mineral Metabolism, 1 (2015) 1-13.

[2] E.U. Alt, C. Senst, S.N. Murthy, D.P. Slakey, C.L. Dupin, A.E. Chaffin, P.J. Kadowitz, R. Izadpanah, Aging alters tissue resident mesenchymal stem cell properties, Stem Cell Research, 8 (2012) 215-225.

[3] C. Szpalski, M. Barbaro, F. Sagebin, S.M. Warren, Bone tissue engineering: Current strategies and techniques - Part II: Cell Types, Tissue engineering Part B, 18 (2012) 258-269.

[4] C.T. Gomillion, K.J. Burg, Stem cells and adipose tissue engineering, Biomaterials, 27 (2006) 6052-6063.

[5] F.H. Shen, B.C. Werner, H. Liang, H. Shang, N. Yang, X. Li, A.L. Shimer, G. Balian, A.J. Katz, Implications of adipose-derived stromal cells in a 3D culture system for osteogenic differentiation: An in vitro and in vivo investigation, Spine J, 13 (2013) 32-43.

[6] J. Rouwkema, N.C. Rivron, C.A. van Blitterswijk, Vascularization in tissue engineering, Trends Biotechnol, 26 (2008) 434-441.

[7] A.E. Mercado-Pagan, A.M. Stahl, Y. Shanjani, Y. Yang, Vascularization in bone tissue engineering constructs, Ann Biomed Eng, 43 (2015) 718-729.

[8] Z.S. Patel, S. Young, Y. Tabata, J.A. Jansen, M.E. Wong, A.G. Mikos, Dual delivery of an angiogenic and an osteogenic growth factor for bone regeneration in a critical size defect model, Bone, 43 (2008) 931-940.

[9] Y. Wang, Y. Wei, X. Zhang, M. Xu, F. Liu, Q. Ma, Q. Cai, X. Deng, PLGA/PDLLA core-shell submicron spheres sequential release system: Preparation, characterization and promotion of bone regeneration in vitro and in vivo, Chemical Engineering Journal, 273 (2015) 490-501. 
[10] L.F. Mendes, R.P. Pirraco, W. Szymczyk, A.M. Frias, T.C. Santos, R.L. Reis, A.P. Marques, Perivascular-like cells contribute to the stability of the vascular network of osteogenic tissue formed from cell sheet-based constructs, PloS One, 7 (2012) e41051.

[11] R.P. Pirraco, B. Melo-Ferreira, T.C. Santos, A.M. Frias, A.P. Marques, R.L. Reis, Adipose stem cell-derived osteoblasts sustain the functionality of endothelial progenitors from the mononuclear fraction of umbilical cord blood, Acta Biomaterialia, 9 (2013) 5234-5242.

[12] J. Kim, H.N. Kim, K.T. Lim, Y. Kim, S. Pandey, P. Garg, Y.H. Choung, P.H. Choung, K.Y. Suh, J.H. Chung, Synergistic effects of nanotopography and co-culture with endothelial cells on osteogenesis of mesenchymal stem cells, Biomaterials, 34 (2013) 7257-7268.

[13] R.P. Pirraco, T. Iwata, T. Yoshida, A.P. Marques, M. Yamato, R.L. Reis, T. Okano, Endothelial cells enhance the in vivo bone-forming ability of osteogenic cell sheets, Laboratory Investigation, 94 (2014) 663-673.

[14] S. Mendez-Ferrer, T.V. Michurina, F. Ferraro, A.R. Mazloom, B.D. Macarthur, S.A. Lira, D.T. Scadden, A. Ma'ayan, G.N. Enikolopov, P.S. Frenette, Mesenchymal and haematopoietic stem cells form a unique bone marrow niche, Nature, 466 (2010) 829-834.

[15] P. Bianco, Bone and the hematopoietic niche: a tale of two stem cells, Blood, 117 (2011) 5281 5288.

[16] F.A. Saleh, M. Whyte, P.G. Genever, Effects of endothelial cells on human mesenchymal stem cell activity in a three-dimensional in vitro model, European Cells and Materials, 22 (2011) 242-257. [17] N.B. Thebaud, R. Siadous, R. Bareille, M. Remy, R. Daculsi, J. Amedee, L. Bordenave, Whatever their differentiation status, human progenitor derived - or mature - endothelial cells induce osteoblastic differentiation of bone marrow stromal cells, Journal of Tissue Engineering and Regenerative Medicine, 6 (2012) e51-60.

[18] S.B. Traphagen, I. Titushkin, S. Sun, K.K. Wary, M. Cho, Endothelial invasive response in a coculture model with physically-induced osteodifferentiation, Journal of Tissue Engineering and Regenerative Medicine, 7 (2013) 621-630. 
[19] J. Leszczynska, B. Zyzynska-Granica, K. Koziak, S. Ruminski, M. Lewandowska-Szumiel, Contribution of endothelial cells to human bone-derived cells expansion in coculture, Tissue Engineering Part A, 19 (2013) 393-402.

[20] E.A. Neofytou, E. Chang, B. Patlola, L.M. Joubert, J. Rajadas, S.S. Gambhir, Z. Cheng, R.C. Robbins, R.E. Beygui, Adipose tissue-derived stem cells display a proangiogenic phenotype on 3D scaffolds, Journal of Biomedical Materials Research Part A, 98 (2011) 383-393.

[21] E. Tumarkin, L. Tzadu, E. Csaszar, M. Seo, H. Zhang, A. Lee, R. Peerani, K. Purpura, P.W. Zandstra, E. Kumacheva, High-throughput combinatorial cell co-culture using microfluidics, Integrative Biology, 3 (2011) 653-662.

[22] S.M. Oliveira, R.L. Reis, J.F. Mano, Towards the design of 3D multiscale instructive tissue engineering constructs: Current approaches and trends, Biotechnol Adv, 33 (2015) 842-855.

[23] J. Borges, J.F. Mano, Molecular interactions driving the layer-by-layer assembly of multilayers, Chemical Reviews, 114 (2014) 8883-8942.

[24] R.R. Costa, J.F. Mano, Polyelectrolyte multilayered assemblies in biomedical technologies, Chemical Society Reviews, 43 (2014) 3453-3479.

[25] P.A. Zuk, M. Zhu, P. Ashjian, D.A.D. Ugarte, J.I. Huang, H. Mizuno, Z.C. Alfonso, J.K. Fraser, P. Benhaim, M.H. Hedrick, Human adipose tissue is a source of multipotent stem cells, Molecular Biology of the Cell, 13 (2002) 4279-4295.

[26] M.T. Cerqueira, L.P.d. Silva, T.C. Santos, R.P. Pirraco, V.M. Correlo, R.L. Reis, A.P. Marques, Gellan Gum-hyaluronic acid spongy-like hydrogels and cells from adipose tissue synergize promoting neoskin vascularization, Appl. Mater. Interfaces, 6 (2014) 19668-19679.

[27] C.R. Correia, P. Sher, R.L. Reis, J.F. Mano, Liquified chitosan-alginate multilayer capsules incorporating poly(L-lactic acid) microparticles as cell carriers, Soft Matter, 9 (2013) 2125-2130.

[28] C.R. Correia, R.L. Reis, J.F. Mano, Multilayered hierarchical capsules providing cell adhesion sites, Biomacromolecules, 14 (2013) 743-751.

[29] C.R. Correia, R.P. Pirraco, M.T. Cerqueira, A.P. Marques, R.L. Reis, J.F. Mano, Semipermeable capsules wrapping a multifunctional and self-regulated co-culture microenvironment for osteogenic differentiation, Scientific Reports, 6 (2016) 1-12. 
[30] C.R. Correia, S. Gil, R.L. Reis, J.F. Mano, A closed chondromimetic environment within magnetic-responsive liquified capsules encapsulating stem cells and collagen II/TGF- $\beta 3$ microparticles, Adv. Healthcare Mater., 5 (2016) 1346-1355.

[31] R. Cai, T. Nakamoto, T. Hoshiba, N. Kawazoe, G. Chen, Matrices secreted during simultaneous osteogenesis and adipogenesis of mesenchymal stem cells affect stem cells differentiation, Acta Biomaterialia, 35 (2016) 185-193.

[32] K. Kolind, D. Kraft, T. Bøggild, M. Duch, J. Lovmand, F.S. Pedersen, D.A. Bindslev, C.E. Bünger, M. Foss, F. Besenbacher, Control of proliferation and osteogenic differentiation of human dental-pulp-derived stem cells by distinct surface structures, Acta Biomaterialia 10 (2014) 641-650. [33] P.T. de Oliveira, A. Nanci, Nanotexturing of titanium-based surfaces upregulates expression of bone sialoprotein and osteopontin by cultured osteogenic cells, Biomaterials 25 (2004) 403-413.

[34] M.D. McKee, A. Nanci. Osteopontin: an interfacial extracellular matrix protein in mineralized tissues. Connective Tissue Research 35 (1996) 197-205.

[35] J.E. Davies. In vitro modeling of the bone/implant interface. Anat Rec 245 (1996) 426-445.

[36] S. De Koker, L.J. De Cock, P. Rivera-Gil, W.J. Parak, R. Auzely Velty, C. Vervaet, J.P. Remon, J. Grooten, B.G. De Geest, Polymeric multilayer capsules delivering biotherapeutics, Adv. Drug Delivery Rev., 63 (2011) 748-761.

[37] A.K. Brun-Graeppi, C. Richard, M. Bessodes, D. Scherman, O.W. Merten, Cell microcarriers and microcapsules of stimuli-responsive polymers, J. Controlled Release, 149 (2011) 209-224.

[38] R.M. Hernandez, G. Orive, A. Murua, J.L. Pedraz, Microcapsules and microcarriers for in situ cell delivery, Advanced Drug Delivery Reviews, 62 (2010) 711-730.

[39] L.J. De Cock, S. De Koker, B.G. De Geest, J. Grooten, C. Vervaet, J.P. Remon, G.B. Sukhorukov, M.N. Antipina, Polymeric multilayer capsules in drug delivery, Angew. Chem., 49 (2010) 69546973.

[40] M. Marguet, C. Bonduelle, S. Lecommandoux, Multicompartmentalized polymeric systems: Towards biomimetic cellular structure and function, Chemical Society Reviews, 42 (2013) 512-529. [41] R.K. Jain, P. Au, J. Tam, D.G. Duda, D. Fukumura, Engineering vascularized tissue, Nature Biotechnol., 23 (2005) 821-823. 
[42] J. Kang, J. Gimble, D. Kaplan, In vitro 3D model for human vascularized adipose tissue, Tissue Engineering Part A, 15 (2009) 2227-2236.

[43] F. Verseijden, H. Jahr, S.P.-v. Sluijs, T.T. Hagen, S. Hovius, A. Seynhaeve, J.v. Neck, G.v. Osch, S. Hofer, Angiogenic capacity of human adipose-derived stromal cells during adipogenic differentiation: An in vitro study, Tissue Engineering Part A, 15 (2009) 445-452.

[44] J. Rehman, D. Traktuev, J. Li, S. Merfeld-Clauss, C.J. Temm-Grove, J.E. Bovenkerk, C.L. Pell, B.H. Johnstone, R.V. Considine, K.L. March, Secretion of angiogenic and antiapoptotic factors by human adipose stromal cells, Circulation, 109 (2004) 1292-1298.

[45] S. Facca, C. Cortez, C. Mendoza-Palomares, N. Messadeq, A. Dierich, A.P. Johnston, D. Mainard, J.C. Voegel, F. Caruso, N. Benkirane-Jessel, Active multilayered capsules for in vivo bone formation, Proc. Nat. Acad. Sci. U. S. A., 107 (2010) 3406-3411.

[46] J. Rouwkema, J.d. Boer, C.A.v. Blitterswijk, Endothelial cells assemble into a 3-dimensional prevascular network in a bone tissue engineering construction, Tissue Engineering, 12 (2006) 26852693. 


\section{Figure legends}

Scheme 1 - Schematic representation of the experimental design. Capsules encapsulating only adipose stem cells (MONO capsules) or adipose stem cells and endothelial cells (CO capsules) were produced (day -21) and cultured in vitro in osteogenic differentiation medium for 21 days. At the day of implantation (day 0), MONO, CO and capsules without cells (MATERIAL) were freshly prepared. Capsules were implanted in nude mice up to 6 weeks. (A) Three capsules of the same formulation were implanted at each pocket. Scale bar is $1 \mathrm{~cm}$. (B) After 1, 3, and 6 weeks, implants were retrieved. The localization of the implanted capsules could be macroscopically visualized. Scale bar is $1 \mathrm{~cm}$.

Figure 1 - Confocal images of (A) MONO and (B) CO capsules encapsulating cells at day 0 previously labelled with DIO (green, ASCs) and DIL (red, ECs) lipophilic dyes. Scale bar is $200 \mu \mathrm{m}$. (C) Osteopontin immunofluorescence (green) in MONO and (D) CO capsules cultured in osteogenic medium for 21 days. Cells nuclei were counterstained with DAPI (blue). Scale bar is $200 \mu$ m. (E) Alizarin red staining on histological sections of MONO and (F) CO capsules cultured in osteogenic differentiation medium for 21 days. Calcium deposits were stained in red. Scale bar is $40 \mu \mathrm{m}$. (G) DNA quantification and $(\mathrm{H})$ ALP activity of MONO and CO capsules freshly prepared at the day of implantation ( 0 days of in vitro culture) or after 21 days in osteogenic differentiation medium (21 days of in vitro culture). Four capsules per well were tested $(n=5)$. Boxplots are presented in Spear style.

Figure 2 - H\&E staining of representative sections from MONO and CO capsules (implantation at day 0 and day 21) and from controls, namely capsules without cells (Material) and pockets without capsules (Empty). Explants were retrieved after 1, 3, and 6 weeks of implantation (scale bar: $50 \mu m$ ). Abbreviations and signs used: microparticles (>), residual material of the liquified environment of capsules $(\$)$, layer-by-layer membrane $(+)$, blood vessels $\left({ }^{*}\right)$ and regions of adipose tissue (AT) are marked. 
Figure 3 - CD31 immunohistochemistry of representative sections from MONO and CO capsules (implantation at day 0 and after 21 days of in vitro culture). The presence of blood vessels is evidenced with squares. Explants were analysed after 6 weeks of implantation. Scale bar is $40 \mu \mathrm{m}$.

Figure 4 - Masson's trichrome staining of representative sections from MONO and CO capsules (implantation at day 0 and after 21 days of in vitro culture). Explants were analysed after 6 weeks of implantation. Collagen is stained in blue. Scale bar is $40 \mu \mathrm{m}$.

Figure 5 - Osteopontin (green) immunohistochemistry counterstained with DAPI (blue) of representative sections from MONO can CO capsules (implantation at day 0 and day 21) and, as control, from capsules without encapsulated cells (Material). Explants were analysed after 1, 3, and 6 weeks of implantation. The insets represent the percentage and the respective standard deviation $(\mathrm{n}=4)$ of green fluorescence quantified by Image $\mathrm{J}$. Scale bar is $50 \mu \mathrm{m}$.

Figure 6 - Alizarin red staining of representative sections from MONO and CO capsules of representative sections from MONO and CO capsules (implantation at day 0 and day 21) and, as control, from capsules without encapsulated cells (Material). Explants were analysed after 1, 3, and 6 weeks of implantation. Mineralization spots are stained in red. Insets represent the percentage and the respective standard deviation $(n=4)$ of red intensity quantified by Image J. Scale bar is 20 $\mu \mathrm{m}$. 

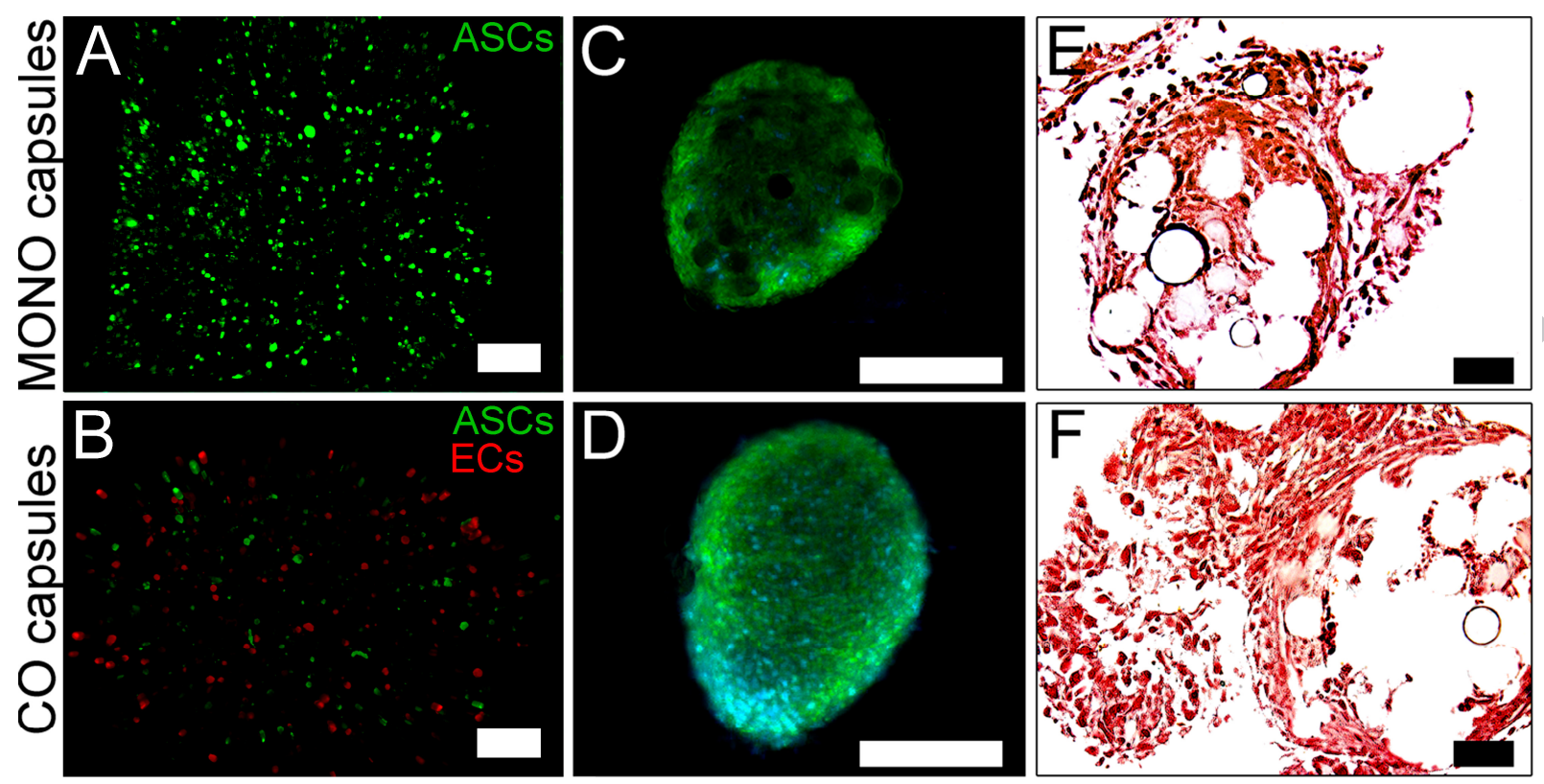

G

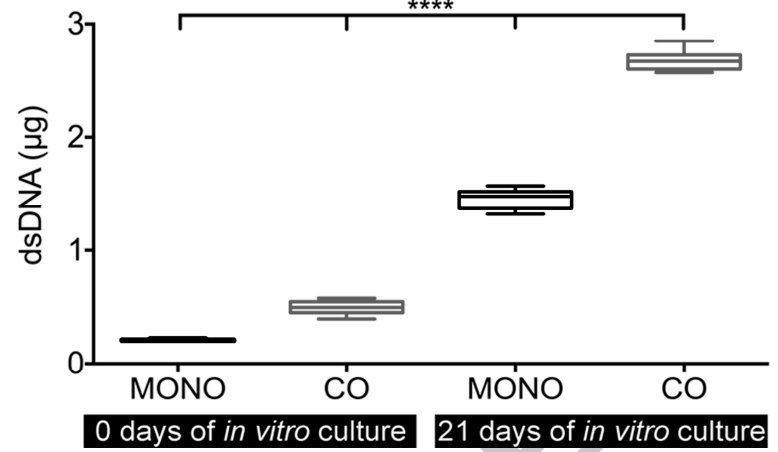

$\mathrm{H}$

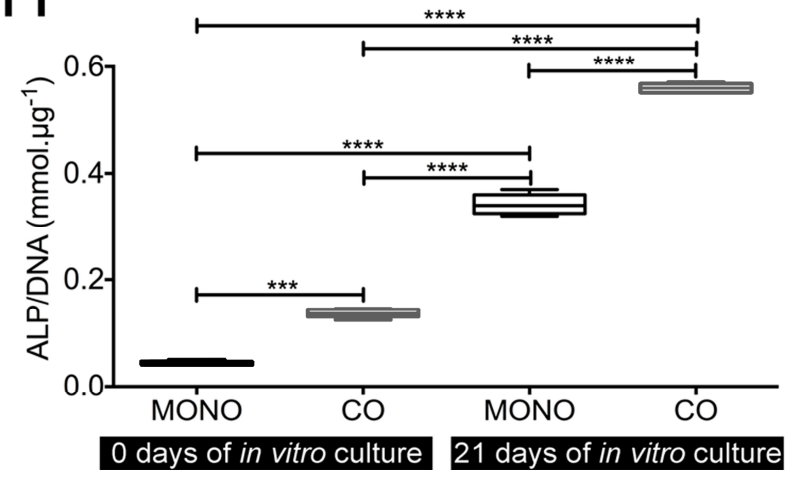

Fig. 1 


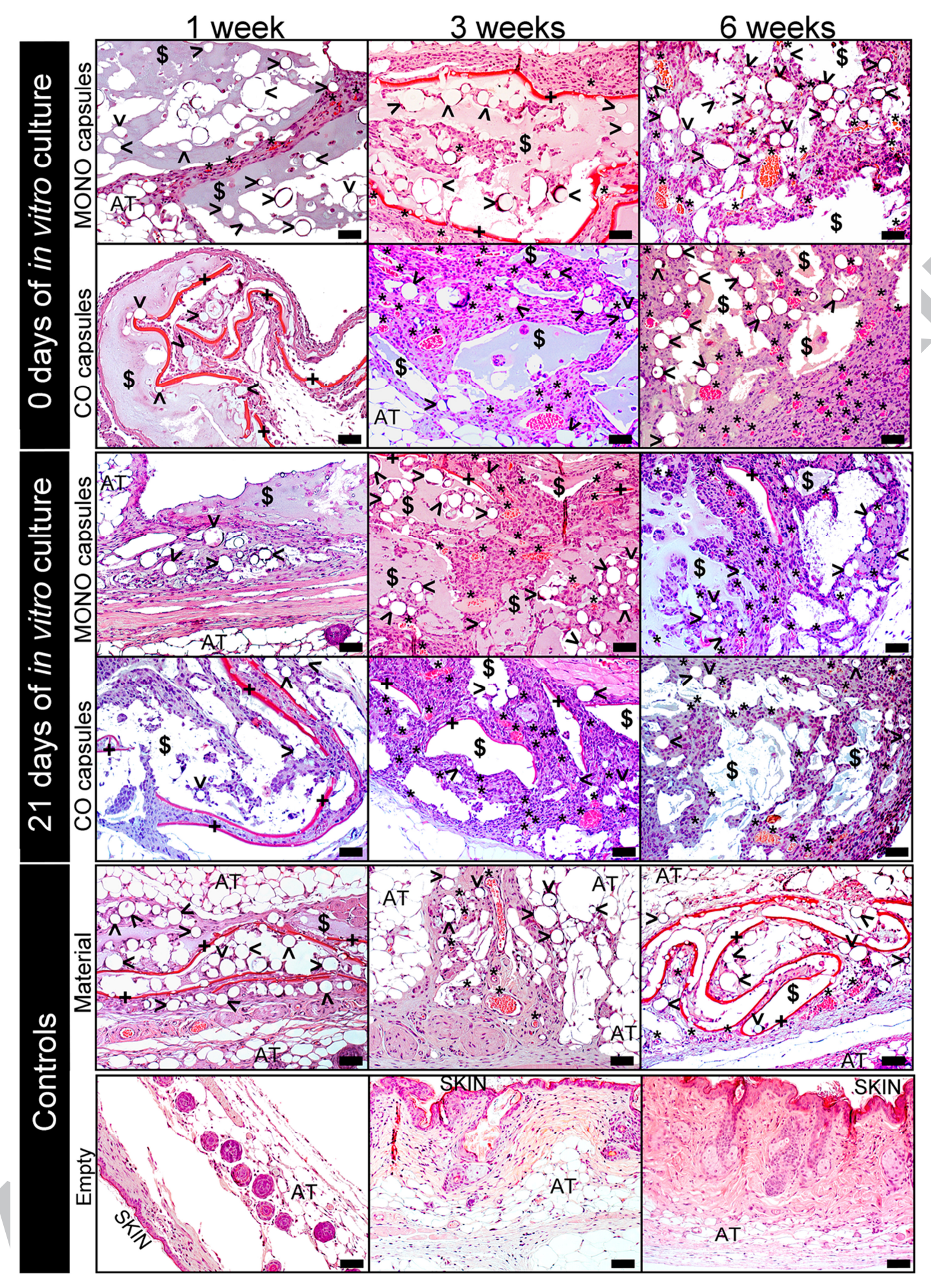

Fig. 2 
MONO capsules CO capsules

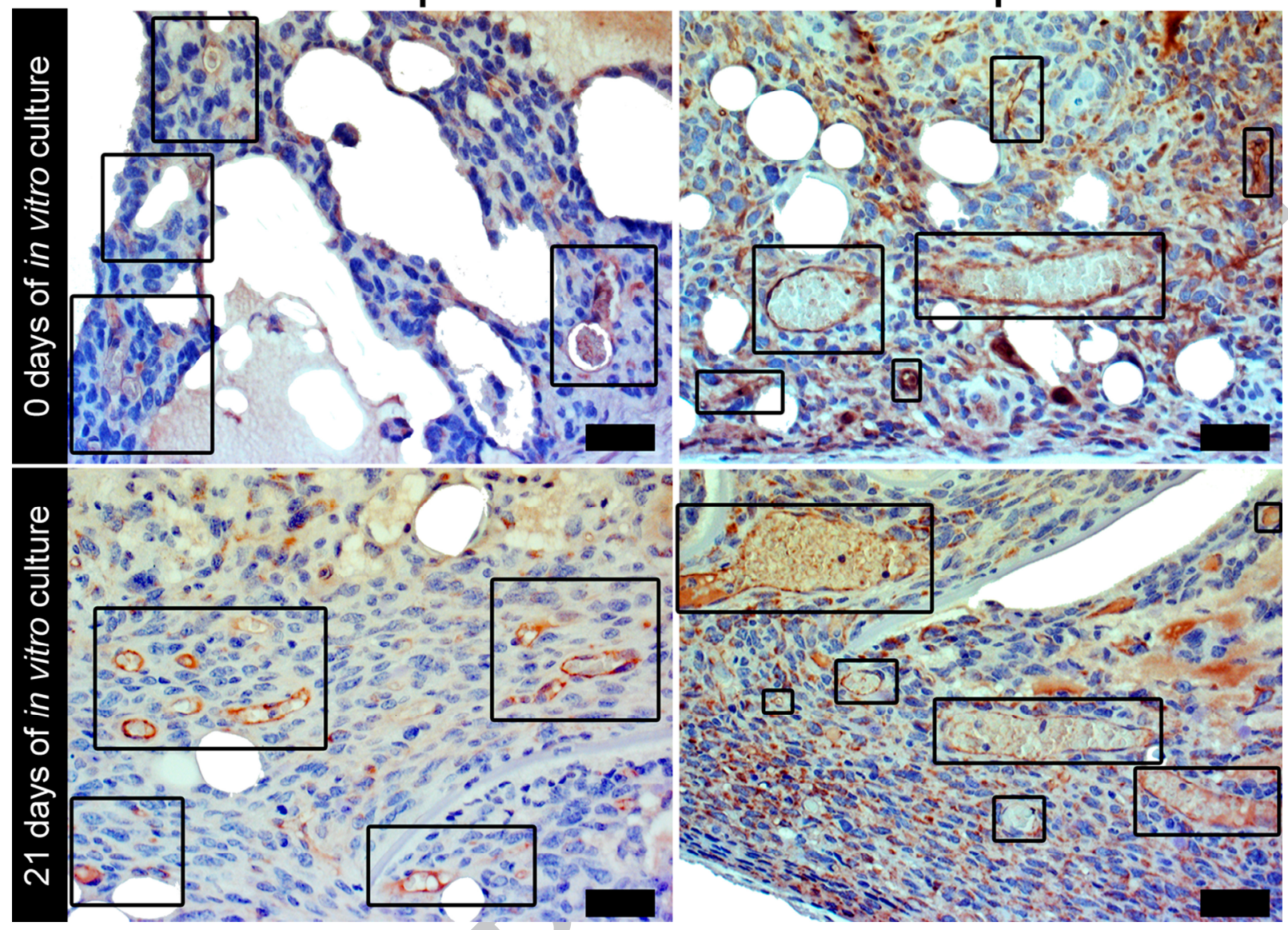

Fig. 3 


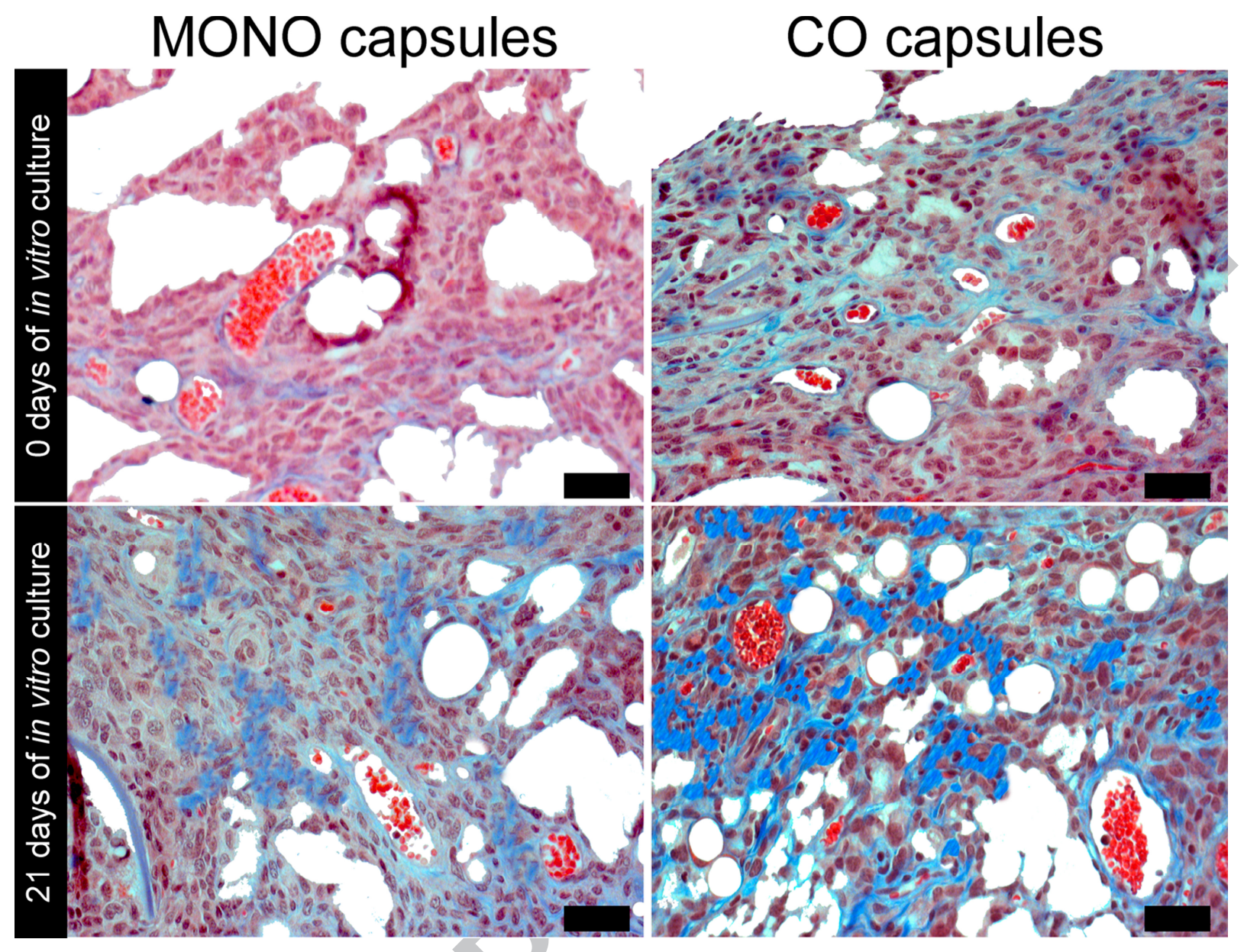

Fig. 4

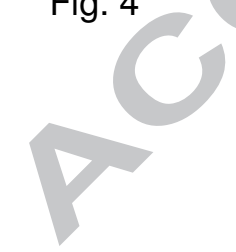


1 week

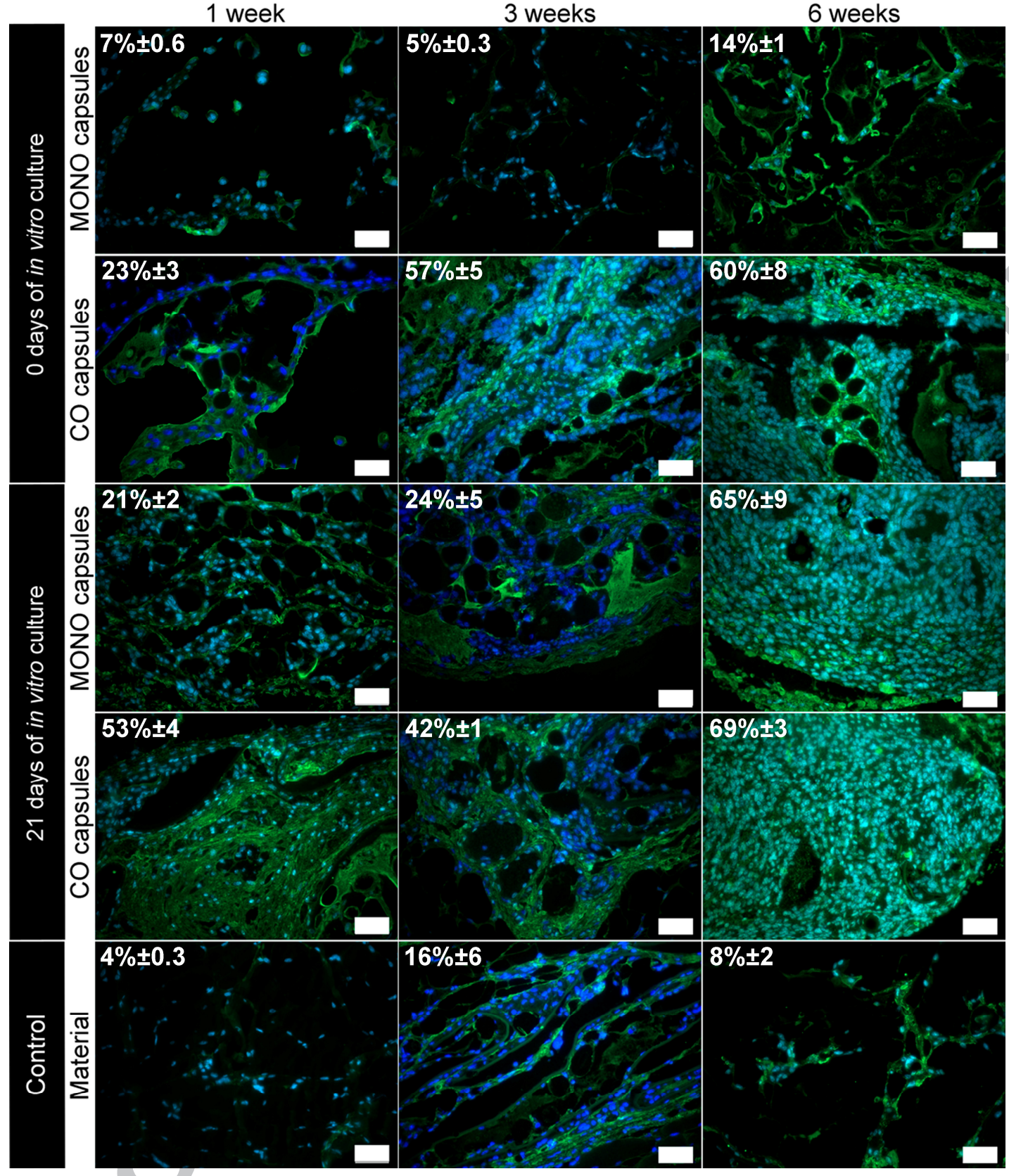

Fig. 5 


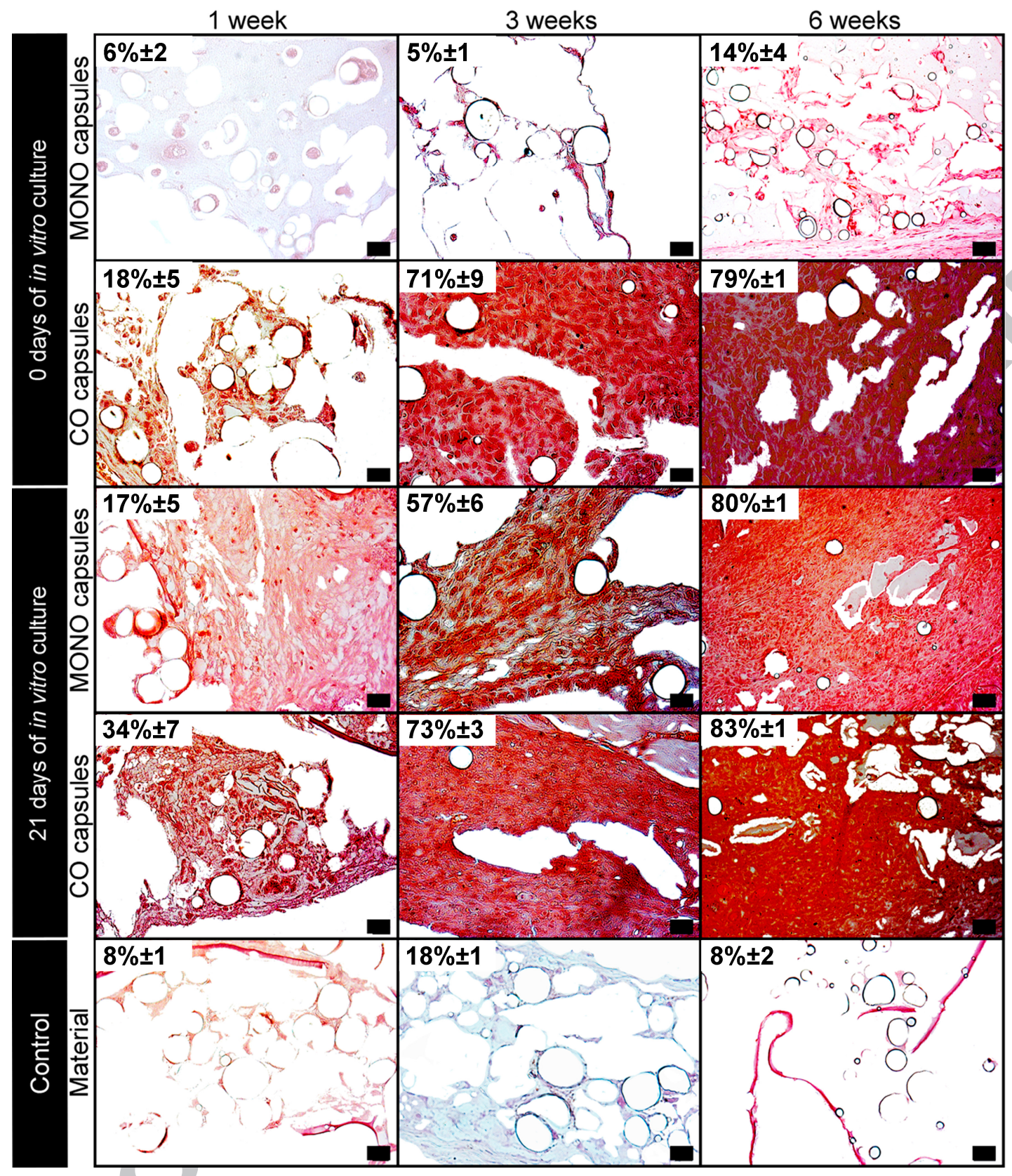

Fig. 6 


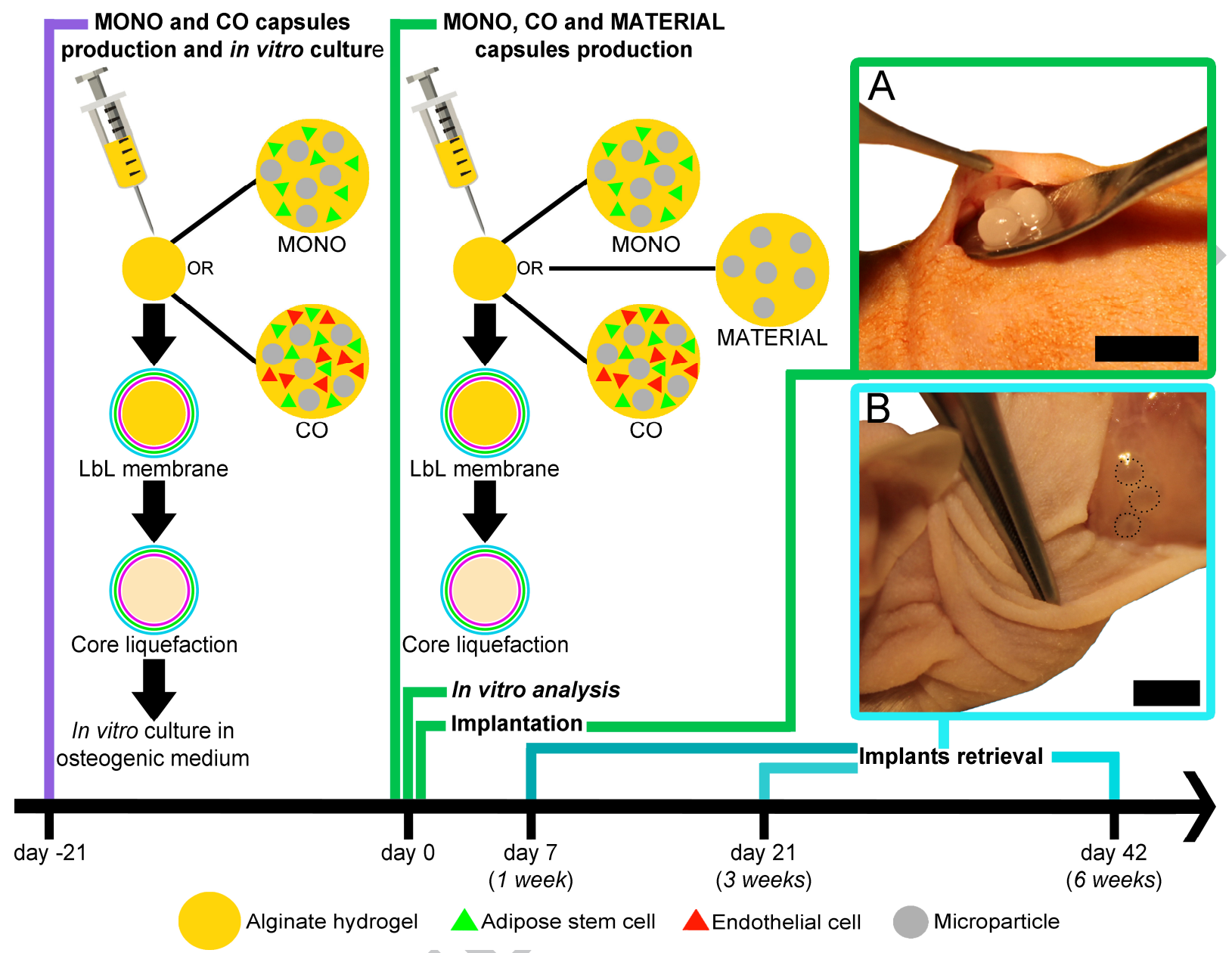

Scheme 1 


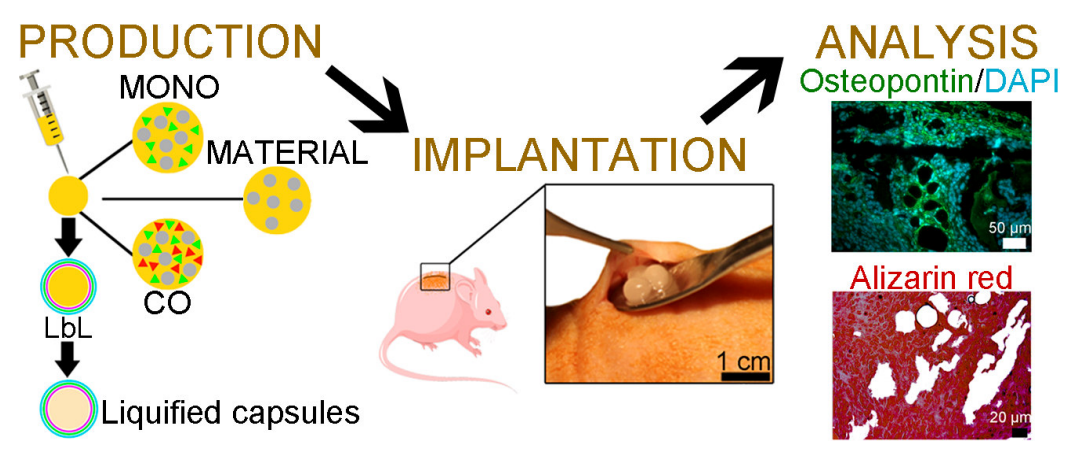

Graphical abstract 


\section{Statement of Significance}

The diffusion efficiency of essential molecules for cell survival is a main issue in cell encapsulation. Former studies reported the superior biological outcome of encapsulated cells within liquified systems. However, most cells used in TE are anchorage-dependent, requiring a solid substrate to perform main cellular processes. We hypothesized that liquified capsules encapsulating microparticles are a promising attempt. Inspired by the multiphenotypic cellular environment of bone, we combine the concept of liquified capsules with co-cultures of stem and endothelial cells. After implantation, results show that co-cultured capsules without in vitro stimulation were able to form a mineralized tissue in vivo. We believe that the present ready-to-use TE strategy requiring minimum in vitro manipulation will find great applicability in bone tissue engineering. 